\title{
Silencing of miR-19a-3p enhances osteosarcoma cells chemosensitivity by elevating the expression of tumor suppressor PTEN
}

\author{
BO ZHANG ${ }^{1}$, YUAN LIU ${ }^{2}$ and JIANGNAN ZHANG ${ }^{1}$ \\ ${ }^{1}$ Department of Orthopaedics, The First People's Hospital of Wenling; ${ }^{2}$ Department of Medical Examination, \\ Center for Disease Control and Prevention of Wenling, Wenling, Zhejiang 317500, P.R. China
}

Received November 27, 2017; Accepted May 16, 2018

DOI: $10.3892 / 01.2018 .9592$

\begin{abstract}
MicroRNAs (miRNAs/miRs) are small non-coding RNAs, which serve important roles in tumor progression. The present study analyzed the role of miR-19a-3p in the chemosensitivity of osteosarcoma (OS) cells. Overexpression of miR-19a-3p was observed in OS cells and a cisplatin-resistant MG63 cell line was subsequently constructed. It was observed that miR-19a-3p inhibitor transfection suppressed cell proliferation and decreased the expression of Ki67 and PCNA compared with the cisplatin treatment group. miR-19a-3p inhibitor transfection also promoted apoptotic rate, increased the expression of Bcl-2 associated $\mathrm{X}$, apoptosis regulator (Bax) and markedly decreased the expression of $\mathrm{Bcl}-2$ compared with the cisplatin treatment group. These results elucidated that silencing of miR-19a-3p enhanced chemosensitivity of OS cells to Cisplatin, through suppressing cell proliferation and promoting cell apoptosis during treatment with Cisplatin. Bioinformatics study and luciferase reporter assays indicated that PTEN was a target of miR-19a-3p, and western blotting demonstrated that PTEN expression was negatively regulated by miR-19a-3p in OS cells. In addition, overexpression of PTEN decreased cell proliferation, but increased apoptotic rate compared with the cisplatin treatment group. It was observed that inhibition of PTEN by $\mathrm{BpV}(\mathrm{HOpic})$ upregulated cell proliferation and downregulated apoptotic rate compared with the Cisplatin-treated miR-19a-3p inhibitor group, indicating that inhibition of PTEN expression
\end{abstract}

Correspondence to: Dr Jiangnan Zhang, Department of Orthopaedics, The First People's Hospital of Wenling, 333 South Chuan'an Road, Wenling, Zhejiang 317500, P.R. China

E-mail: zhangjiangnanwl@163.com

Abbreviations: OS, osteosarcoma; miRNAs, microRNAs; Bax, Bcl-2 associated $\mathrm{X}$, apoptosis regulator; 3'-UTR, 3'-untranslated region; PTEN, phosphatase and tensin homolog; BMSCs, bone marrow derived stroma cells; RT-qPCR, reverse transcription-quantitative polymerase chain reaction; CCK-8, Cell Counting Kit-8

Key words: miR-19a-3p, osteosarcoma, chemosensitivity, PTEN counteracted the effect of the miR-19a-3p inhibitor on the regulation of chemosensitivity in OS cells. Taken together, overexpression of miR-19a-3p was observed in OS cell lines and that downregulation of miR-19a-3p enhanced the chemosensitivity of OS cells to Cisplatin, by elevating the expression of the tumor suppressor, PTEN.

\section{Introduction}

Osteosarcoma (OS) is one of the most commonly diagnosed types of malignant bone cancer in adolescents and young adults (1). The long-term disease-free survival of patients with OS remains low $(\sim 65 \%)$ and disease-free survival of patients exhibiting metastasis have a lower survival rate (2). Due to the improvement of chemotherapy drugs, the 5 -year survival rate of OS patients has increased in recent decades (3). However, chemoresistance remains a major problem, and contributes to the low 5-year survival rate (4). Previous studies have indicated that various oncogenes and tumor suppressors are involved in the chemoresistance of OS cells $(5,6)$. However, the existing understanding of the mechanism of chemoresistance in OS is limited and further investigation is urgently required.

MicroRNAs (miRNAs/miRs), a group of small non-coding RNAs that regulate gene expression by binding to the 3'-untranslated region (3'-UTR) of mRNAs, serve important roles in various cellular processes, including proliferation, apoptosis, invasion and differentiation $(7,8)$. miRNAs are dysregulated in various types of cancer, acting as oncogenes or tumor suppressors during tumorigenesis (9). Evidence demonstrated that miRNA serves a regulatory role in the chemosensitivity of various types of cancer cells. miRNA-107 enhanced chemosensitivity to paclitaxel in non-small cell lung cancer by targeting the antiapoptotic factor Bcl-2 (10). miRNA-625 has been demonstrated to increase chemosensitivity in glioma by directly targeting AKT serine/threonine kinase 2 AKT2 (11). The aim of the present study was to elucidate the regulatory role of miR-19a-3p in the chemosensitivity of OS cells. miR-19a-3p was reported to enhance proliferation and insulin secretion, while inhibiting the apoptosis of pancreatic $\beta$-cells through the inhibition of suppressor of cytokine signaling 3 (SOCS3) (12). However, the role of miR-19a-3p 
in the regulation of chemosensitivity is unclear and requires further examination.

Down-regulation of phosphatase and tensin homolog (PTEN), a tumor suppressor gene, was reported in various human primary tumors (13). Previous studies have also demonstrated that PTEN was associated with chemosensitivity of cancer cells. The study of Shen et al (14) indicated that downregulation of miRNA-147 expression increased the chemosensitivity of gastric cancer cells to 5-fluorouracil by directly targeting PTEN. Using bioinformatics analysis, it was revealed in the present study that PTEN was a putative target gene of miR-19a-3p. Accordingly, the hypothesis was that miR-19a-3p may be involved in the regulation of chemosensitivity through targeting PTEN in OS cells.

In the present study, overexpression of miR-19a-3p was identified in OS cells and that silencing of miR-19a-3p enhanced the chemosensitivity of OS cells by elevating the expression of PTEN. These results will assist in the understanding of the underlying mechanism of involvement of miR-19a-3p in regulating chemosensitivity of OS cells.

\section{Materials and methods}

Cell culture and induction of cisplatin resistant cells. Bone marrow-derived stroma cells (BMSCs) were purchased from BeNa Culture Collection (Bejing, China) and the OS cell lines, MNNG/HOS, U-2 OS, MG63, Saos-2, were purchased from the American Type Culture Collection (Manassas, VA, USA). Cells were cultured in DMEM (Invitrogen; Thermo Fisher Scientific, Inc., Waltham, MA, USA) supplemented with $10 \%$ fetal bovine serum (Gibco; Thermo Fisher Scientific, Inc.) at $37^{\circ} \mathrm{C}$ in $5 \% \mathrm{CO}_{2}$. Cisplatin was purchased from Sigma-Aldrich (Merck KGaA, Darmstadt, Germany) and dissolved in PBS. For the induction of cisplatin-resistant cells, MG63 cells were treated by gradually increasing doses of cisplatin in the cell culture medium throughout the passages for $6 \sim 8$ months. The cells were maintained in the presence of $5 \mu \mathrm{M}$ cisplatin in the culture medium for $48 \mathrm{~h}$ in every alternate passage. Bpv(HOpic) (Merck KGaA, Darmstadt, Germany), a PTEN inhibitor, was used to treat cells at a concentration of $1 \mu \mathrm{M}$.

Reverse transcription-quantitative polymerase chain reaction $(R T-q P C R)$. The expression level of miR-19a-3p in the cell lines was measured via RT-qPCR. Total RNA was extracted from the cells using the TRIzol (Invitrogen; Thermo Fisher Scientific, Inc.), according to the manufacturer's protocol. cDNA was synthesized using a miScript reverse transcription kit (Qiagen, GmbH, Hilden, Germany) and the PCR reaction was performed using the SYBR Premix Ex Taq ${ }^{\mathrm{TM}}$ II kit (Takara Bio, Inc., Otsu, Japan), according to the manufacturer's instructions. The primers used were as following, miR-19a-3p forward, 5'-GGGGGGGTGTGCAAATCT-3', and reverse, 5'-GTGCGTGTCGTGGAGTCG-3'; U6, forward, 5'-GCT TCGGCAGCACATATACTAAAAT-3', and reverse, 5'-CGC TTCACGAATTTGCGTGTCAT-3'. The amplification protocol included an initial denaturation step at $95^{\circ} \mathrm{C}$ for $10 \mathrm{~min}$, followed by 40 cycles of $95^{\circ} \mathrm{C}$ for $15 \mathrm{sec}$ and $60^{\circ} \mathrm{C}$ for $60 \mathrm{sec}$. The expression levels were calculated using the $2^{-\Delta \Delta \mathrm{Cq}}$ method with U6 used for normalization (15).
Transfection. Cells were seeded into 96-well plates to reach $60 \%$ confluence for transfection. miR-19a-3p mimics (cat. no. MIMAT0000073) and inhibitor (cat. no. MIMAT0021837) were purchased from Invitrogen; (Thermo Fisher Scientific, Inc.). According to manufacturer's protocol, transfections were performed using Lipofectamine ${ }^{\circledR} 2000$ (Invitrogen; Thermo Fisher Scientific, Inc.), according to the manufactuerer's protocol. For overexpression of PTEN, the full-length PTEN sequence (5'-UUCACAUCCUACCCCUUUGCACU-3') obtained from Invitrogen (Thermo Fisher Scientific, Inc.) was cloned into a pcDNA3.0 vector (Invitrogen; Thermo Fisher Scientific, Inc). Cells were transfected with pcDNA3.0-PTEN using Lipofectamine ${ }^{\circledR} 2000$ (Invitrogen; Thermo Fisher Scientific, Inc.), according to the manufactuerer's protocol. After transfection for $48 \mathrm{~h}$, cells were collected for subsequent experimentation.

Cell Counting Kit-8 (CCK-8) assay. Cells were seeded at $5 \times 10^{3}$ per well in 96-well plates and incubated for 0-5 days with and without cisplatin treatment $(3 \mu \mathrm{M})$. A total of $10 \mu \mathrm{l}$ of CCK-8 solution (Beyotime Institute of Biotechnology, Shanghai, China) was incubated at $37^{\circ} \mathrm{C}$ for $2 \mathrm{~h}$. The absorbance was measured at $450 \mathrm{~nm}$ using a microplate spectrophotometer (Molecular Devices, LLC, Sunnyvale, CA, USA). Triplicate wells were used in each group.

Western blotting. According to the manufacturer's protocol, proteins were extracted from cells using RIPA lysis buffer (Beyotime Institute of Biotechnology). Homogenized samples were washed with ice-cold PBS and centrifuged at 10,000 x g for $15 \mathrm{~min}$ at $4^{\circ} \mathrm{C}$. The supernatant was collected and the protein concentration was determined using a BCA protein assay kit (Beijing Solarbio Science \& Technology Co., Ltd., Beijing, China). A total of $20 \mu \mathrm{g}$ protein were loaded per lane and separated by $10 \%$ SDS-PAGE and then transferred into PVDF membranes (Merck KGaA). The membrane was blocked with $5 \%$ bovine serum albumin for $1 \mathrm{~h}$ at room temperature and then incubated with the following primary monoclonal antibodies (at a 1:1,000 dilution): Anti-Ki67 (cat. no. ab92742; Abcam, Cambridge, UK), anti-PCNA (cat. no. 2586; Cell Signaling Technology, Inc., Danvers, MA, USA), anti-Bax (cat. no. ab32503; Abcam), anti-Bcl-2 (cat. no. ab32124; Abcam), anti-PTEN (cat. no. ab79156; Abcam) and GAPDH (cat. no. 5174; Cell Signaling Technology, Inc.) and gently agitated at $4^{\circ} \mathrm{C}$ overnight. Then the membranes were incubated with horseradish peroxidase-conjugated secondary antibodies (Anti-rabbit IgG, HRP-linked Antibody, cat. no. 7074; Cell Signaling Technology, Inc.; dilution 1:3,000) for $1 \mathrm{~h}$ at room temperature. An enhanced chemiluminescence system (Bio-Rad Laboratories, Inc., Hercules, CA, USA) was used for the detection of antibody-bound proteins, according to manufacturer's instructions. Analysis was performed using ImageJ software (version 1.48, National Institutes of Health, Bethesda, MD, USA).

Cell apoptosis assay. An Annexin V-FITC/propidium iodide (PI) apoptosis detection kit (Multisciences, Shanghai, China, http://liankebio.biomart.cn/) was used for the cell apoptosis assay, according to manufacturer's protocol. A total of 
$3 \times 10^{5}$ cells were washed with PBS, resuspended and incubated with $5 \mu 1$ Annexin V-FIFC and $10 \mu 1$ PI. Cell apoptosis was analyzed using a flow cytometer (BD Biosciences, Franklin Lakes, NJ, USA). FACS data was processed using FlowJo (version 7.5.5; Tree Star Inc., Ashland, OR, USA).

MiRNA target prediction. Genes containing the binding sites of the miR-19a-3p 3'-UTR were obtained using TargetScan (http://www.targetscan.org/vert_71/), MiRanda (http://www. microrna.org/microrna/home.do), PICTAR (http://www.pictar. org/) and miRDB (http://mirdb.org/miRDB/custom.html) target prediction algorithms.

Luciferase reporter assay. The fragment of PTEN containing the target sequence 5'-UUUGCAC-3', Invitrogen, Thermo Fisher Scientific, Inc.) of miR-19a-3p was cloned into pGL3 Luciferase Reporter Vectors (Promega Corporation, Madison, WI, USA) to form the reporter vector PTEN-wild-type (PTEN WT), while the PTEN-mutated-type (PTEN MUT) contained the mutated binding site. The cells were co-transfected with PTEN WT or PTEN MUT and miR-19a-3p mimics using Lipofectamine ${ }^{\circledR} 2000$, according to manufacturer's protocol. Following transfection for $48 \mathrm{~h}$, luciferase activity was measured using the Dual-Luciferase ${ }^{\circledR}$ Reporter assay system kit (Promega Corporation), according to the manufacturer's instructions. Renilla luciferase activity was used for normalization.

Statistical analysis. All data are presented as the mean \pm standard deviation. Statistical analysis was performed by Student's t-test or one-way analysis of variance with Student-Newman-Keuls post hoc test, using SPSS 19.0 (IBM Corp., Armonk, NY, USA). P $>0.05$ was considered to indicate a statistically significant difference.

\section{Results}

miR-19a-3p is overexpressed in OS cells. To investigate the role of miR-19a-3p in OS, its expression level in OS cells was detected using RT-qPCR. The expression of miR-19a-3p that was revealed in OS cells, MNNG/HOS, U-2 OS, MG63 and Saos-2, was higher than that in normal BMSCs $(\mathrm{P}<0.01$; Fig. 1A). Transfection with miR-19a-3p mimics or inhibitor increased and decreased the expression of miR-19a-3p significantly in $\mathrm{U}-2 \mathrm{OS}$ and MG63 cells, respectively $(\mathrm{P}<0.01$; Fig. 1B). The expression of miR-19a-3p was highest in MG63 cells, thus, MG63 cells were selected for the following experiments.

Inhibition of miR-19a-3p enhances chemosensitivity of OS cells. A Cisplatin-resistant MG-63 cell line was constructed, as described in the Materials and methods section. Cisplatin treatment did not significantly affect cell proliferation or apoptosis of cisplatin-resistant MG-63 cells. However, transfection with the miR-19a-3p inhibitor downregulated the rate of cell proliferation and decreased the expression of the proliferation-associated proteins, Ki67 and PCNA. Transfection with miR-19a-3p inhibitor NC did not have a significant effect compared with the Cisplatin group $(\mathrm{P}>0.05$; Fig. 2A-C), suggesting that downregulation of miR-19a-3p suppressed the proliferation of Cisplatin-resistant OS cells under Cisplatin treatment. Transfection with miR-19a-3p inhibitor increased cell apoptotic rate, while miR-19a-3p mimics did not have a significant effect compared with the Cisplatin group ( $\mathrm{P}>0.05$; Fig. 2D and $\mathrm{E})$. Transfection with miR-19a-3p inhibitor increased the expression of Bax and decreased the expression of $\mathrm{Bcl}-2$ compared with the Cisplatin group $(\mathrm{P}<0.05$; Fig. $2 \mathrm{~F}$ and $\mathrm{G})$, suggesting that downregulation of miR-19a-3p expression promoted apoptosis of Cisplatin-resistant OS cells under Cisplatin treatment. The data indicates that miR-19a-3p inhibitor increases the chemosensitivity of OS cells to Cisplatin.

PTEN is a target of miR-19a-3p in OS cells. To explore the chemosensitivity regulatory mechanism of miR-19a-3p in OS cells, the public database, TargetScan was used to search for potential targets of miR-19a-3p. It was revealed that the PTEN mRNA 3'-UTR contained highly conserved sequences, which were targeted by miR-19a-3p (Fig. 3A). Expression of endogenous PTEN protein was detected in OS cells. PTEN expression was revealed to be significantly downregulated in MNNG/HOS, U-2 OS, MG63 and Saos-2 cells compared with BMSCs cells $(\mathrm{P}<0.05, \mathrm{P}<0.01$; Fig. $3 \mathrm{~B}$ and $\mathrm{C})$. Expression of PTEN was downregulated in the miR-19a-3p mimics group and upregulated in the miR-19a-3p inhibitor group, compared with the control group $(\mathrm{P}<0.05$; Fig. 3D and $\mathrm{E})$. To confirm the association between PTEN and miR-19a-3p in OS cells, a luciferase reporter assay was conducted. It was revealed that co-transfection with miR-19a-3p mimics and PTEN WT led to a significant decrease in luciferase activity. However, co-transfection with miR-19a-3p mimics and PTEN MUT did not have a significant effect on luciferase activity $(\mathrm{P}>0.01$; Fig. 3F). In summary, these results indicated that PTEN was a target of miR-19a-3p and that the expression of PTEN was negatively regulated by miR-19a-3p in OS cells.

Overexpressed PTEN enhances the chemosensitivity of OS cells. MG-63 cells were transfected with pcDNA3.0-PTEN to increase the protein expression level of PTEN $(\mathrm{P}<0.05$; Fig. 4A and B). It was demonstrated that overexpressed PTEN decreased cell proliferation and increased apoptotic rate compared with the Cisplatin group ( $\mathrm{P}<0.05$; Fig. $4 \mathrm{C}$ and $\mathrm{D})$. The results indicate that overexpressed PTEN enhances the chemosensitivity of OS cells.

PTEN inhibitor BpV(HOpic) counteracts the effect of miR-19a-3p inhibitor in the regulation of chemosensitivity. To investigate the interaction between PTEN and miR-19a-3p, the PTEN inhibitor, $\mathrm{BpV}$ (HOpic), was used in the present study. It was observed that $\mathrm{BpV}(\mathrm{HOpic})$ treatment increased cell proliferation and decreased apoptotic rate compared with the Cisplatin-treated miR-19a-3p inhibitor group $(\mathrm{P}<0.05$ Fig. $5 \mathrm{~A}$ and $\mathrm{B})$. These results indicate that inhibition of PTEN counteracted the effect of the miR-19a-3p inhibitor on the regulation of chemosensitivity in OS cells.

\section{Discussion}

In the treatment of OS, the combination of chemotherapy and surgery has improved the overall 5-year survival rate of patients with OS (16). Cisplatin is one of the most commonly 

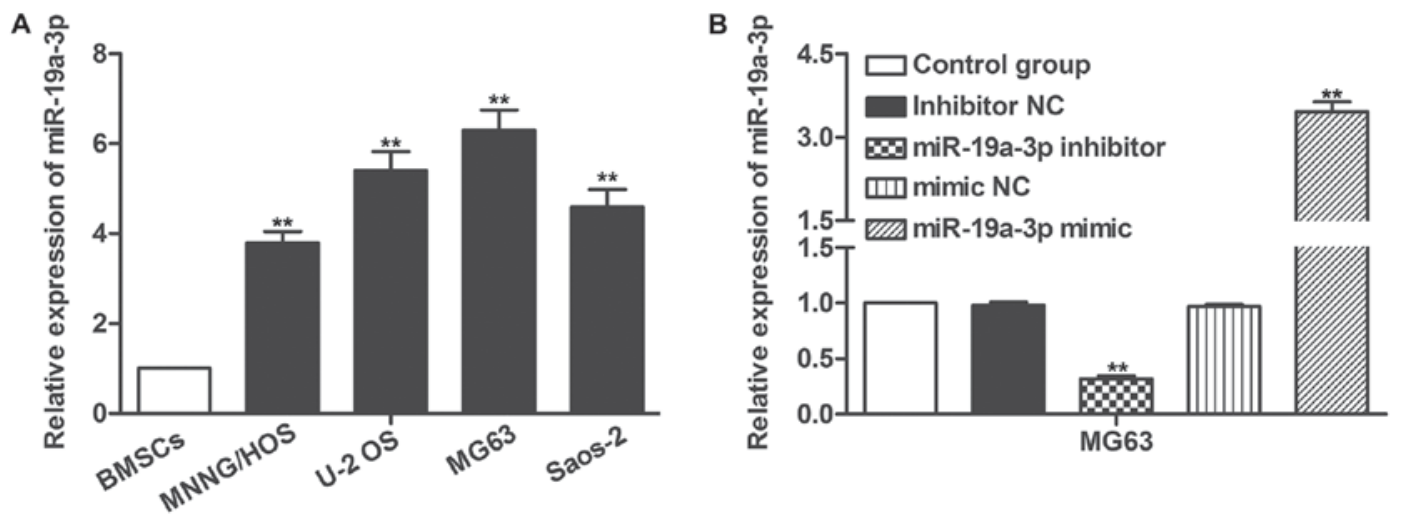

Figure 1. miR-19a-3p is overexpressed in OS cells. (A) The relative expression of miR-19a-3p was compared among BMSCs and OS cell lines by RT-qPCR. ${ }^{* *} \mathrm{P}<0.01$ compared with BMSCs. (B) Relative expression of miR-19a-3p was measured in MG63 cells transfected with miR-19a-3p mimic or inhibitor by RT-qPCR. ${ }^{* *} \mathrm{P}<0.01$ compared with the control group. The error bars represent mean \pm standard deviation of three independent experiments. miR, microRNA; OS, osteosarcoma; BMSCs, bone marrow derived stroma cells; RT-qPCR, reverse transcription-quantitative polymerase chain reaction.
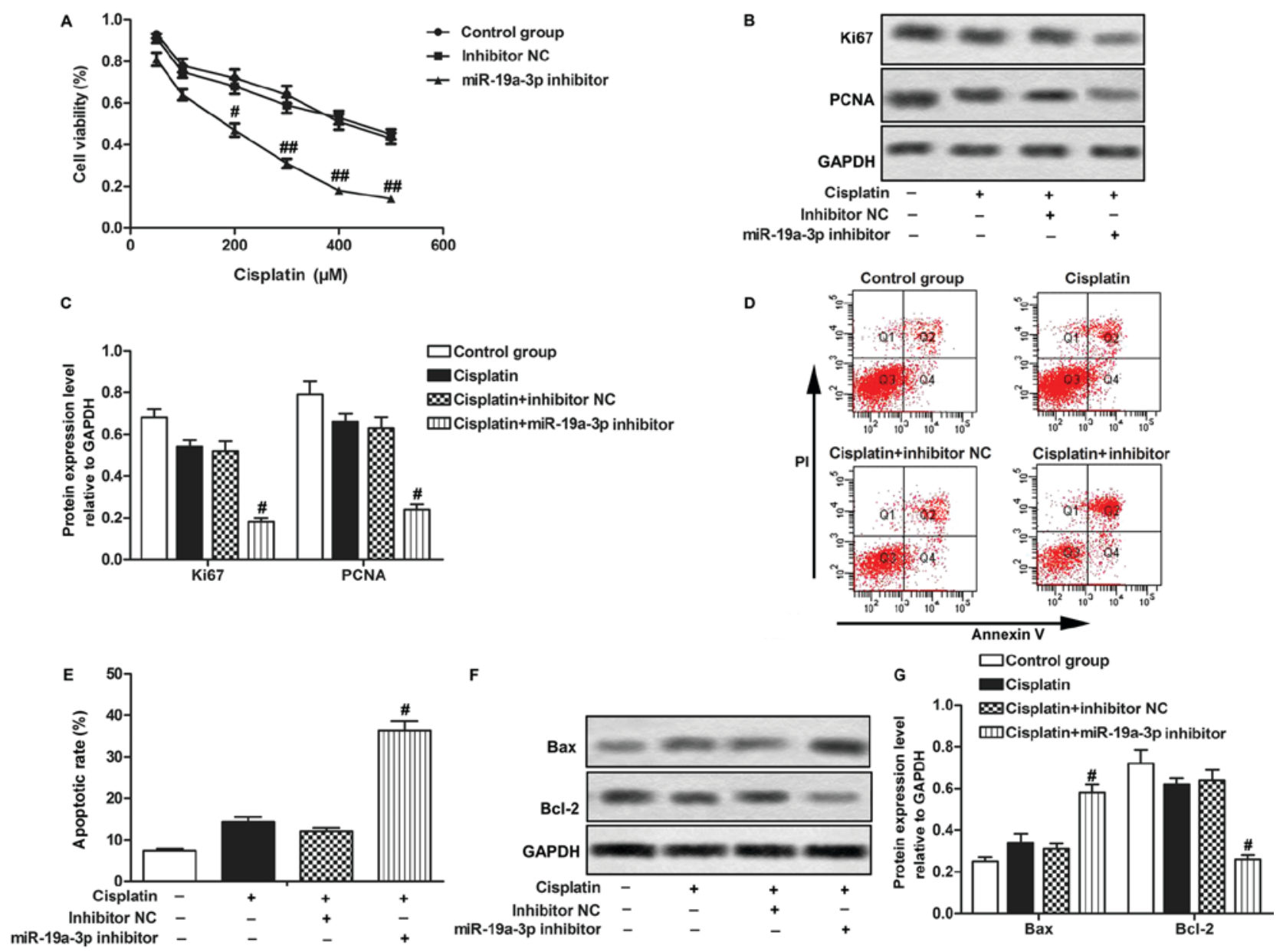

Figure 2. miR-19a-3p inhibitor enhances chemosensitivity of OS cells. A Cisplatin-resistant cell line was constructed, and Cisplatin-resistant cells were transfected with miR-19a-3p mimics or inhibitor. Cisplatin treatment of cisplatin-resistant cells was used as a control. (A) Cell proliferation was measured by CCK-8 assay. (B) Expression of Ki67 and PCNA protein was detected through western blotting. (C) Quantification of the protein expression of Ki67 and PCNA. (D) Apoptosis was detected by flow cytometric analysis. (E) Quantification of cell apoptotic rate. (F) Expression of Bax and Bcl-2 protein was detected through western blotting. (G) Quantification of Bax and Bcl-2 protein expression. The error bars represent means \pm standard deviation of three independent experiments. ${ }^{\#} \mathrm{P}<0.05,{ }^{\# \#} \mathrm{P}<0.01$ compared with Cisplatin group. miR, microRNA; OS, osteosarcoma; CCK-8, Cell Counting Kit-8; PCNA, proliferating cell nuclear antigen; Bax, BCL2 associated $\mathrm{X}$, apoptosis regulator.

used platinum-based anticancer drugs for the treatment of OS, interacting with nucleophilic $\mathrm{N}^{7}$-sites of purine bases to induce DNA damage that leads to cell death (17). However,
Cisplatin resistance can develop and limit the effectiveness of chemotherapeutics. Therefore, understanding the molecular mechanism of chemoresistance to Cisplatin is essential to 
B

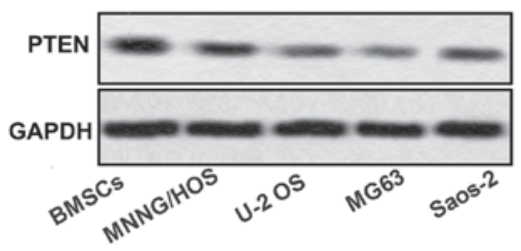

C
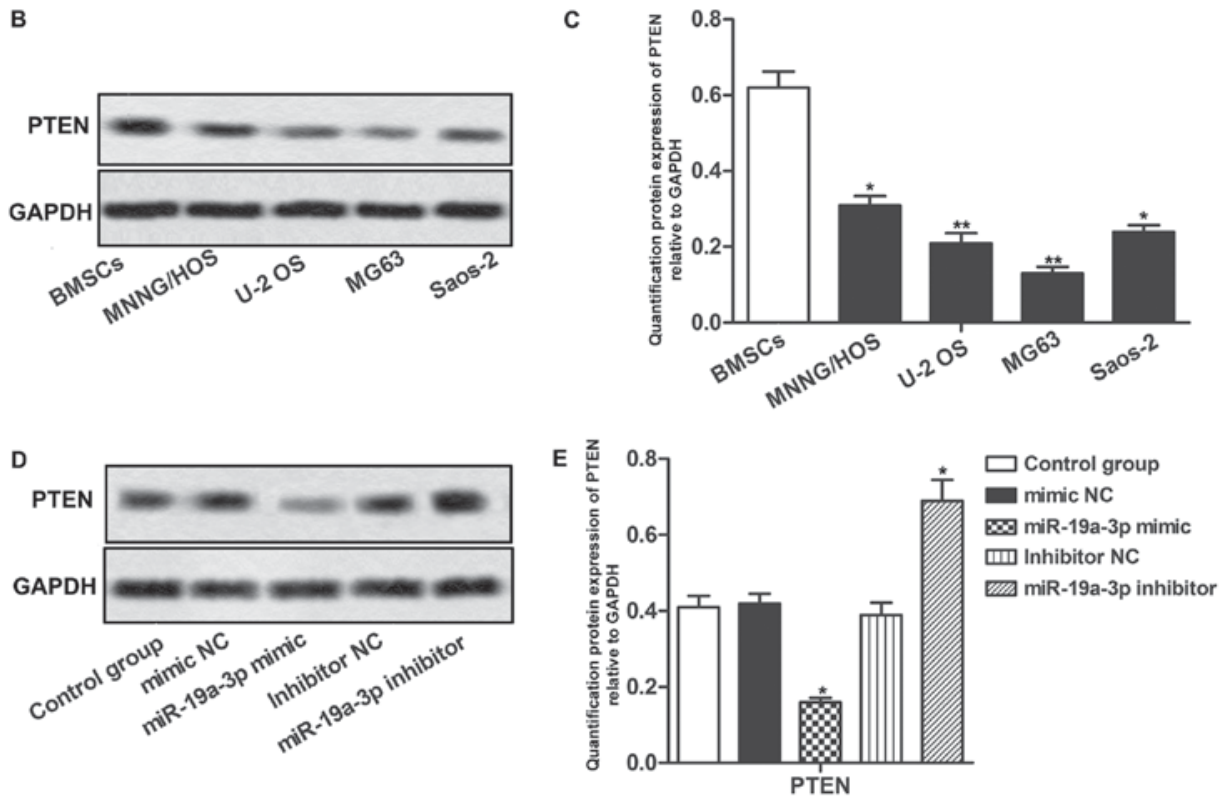

$\mathbf{F}$

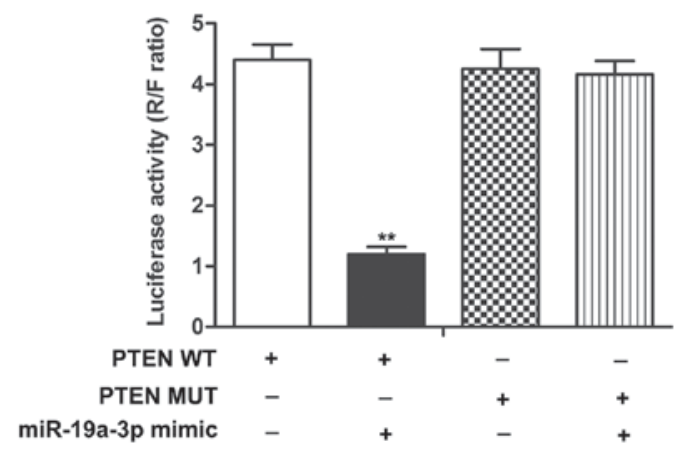

Figure 3. PTEN is a target of miR-19a-3p in OS cells. (A) The putative binding site of miR-19a-3p in 3'-UTR of PTEN. (B) Protein expression of PTEN in OS cell lines and bone marrow-deived stromal cells was measured through western blotting. (C) Quantification of the protein expression of PTEN in MNNG/HOS, U-2 OS, MG63, Saos-2. ${ }^{*} \mathrm{P}<0.05,{ }^{* *} \mathrm{P}<0.01$ compared with BMSCs group. (D) Relative expression of PTEN in MG63 cells transfected with miR-19a-3p mimics or inhibitor was measured through western blotting. (E) Quantification of the protein expression level of PTEN relative to GAPDH. "P<0.05, compared with control group. (F) Results of the luciferase reporter assay. ${ }^{* *} \mathrm{P}<0.01$ compared with PTEN WT group. PTEN, phosphatase and tensin homolog; miR, microRNA; OS, osteosarcoma; 3'-UTR, 3'-untranslated region; BMSC, bone marrow derived stroma cell; WT, wild type; MUT, mutated; NC, negative control; R, Renilla; F, Firefly.

develop a more effective treatment for OS. In the present study, it was demonstrated that silencing of miR-19a-3p enhanced chemosensitivity to Cisplatin in OS cells by up-regulating the expression of PTEN. These results can assist in finding a better therapeutic strategy against Cisplatin resistance in OS.

miRNAs, a class of non-coding regulatory RNAs, have been reported to be involved in tumor progression $(18,19)$. As miRNAs act as oncogenes or tumor suppressor genes, they constitute a large gene network for the regulation of various cellular processes during tumorigenesis (9). Evidence suggests that numerous miRNAs are associated with chemoresistance in various types of cancers. For example, miR-491 has been demonstrated to inhibit chemoresistance of OS cells by targeting $\alpha \mathrm{B}$-crystallin (20). miR-608 has been demonstrated to inhibit gemcitabine chemoresistance by regulating resistance genes in pancreatic cancer cells (21). Previous studies have reported that miR-19a-3p expression was correlated with cell proliferation and autophagy $(12,22)$. In addition, the serum level of miR-19a-3p has been reported to be significantly higher in patients with colorectal cancer compared with healthy patients (23). Similarly, it was demonstrated that the expression of miR-19a-3p was upregulated in OS cells compared with normal BMSCs. In the present study a cispaltin-resistant MG-63 cell line was constructed to investigate the role of miR-19a-3p in the chemosensitivity of OS cells. It was observed that Cisplatin treatment alone did not significantly affect proliferation or apoptosis of cispaltin-resistant MG-63 cells. However, Cisplatin treatment combined with miR-19a-3p inhibitor transfection suppressed cell proliferation and decreased the expression of 
A
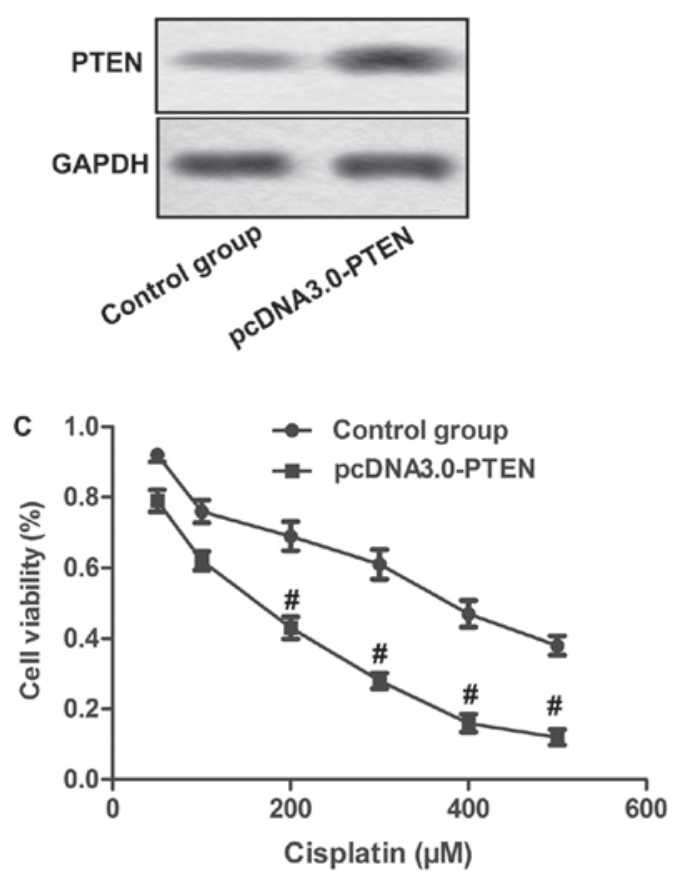
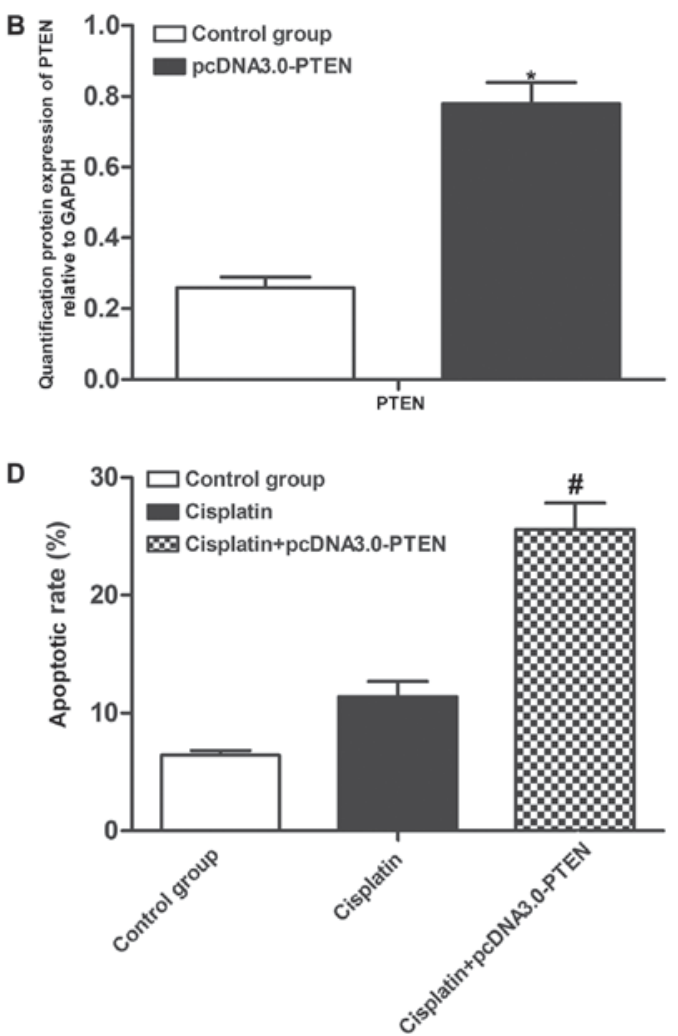

Figure 4. Overexpression of PTEN enhances the chemosensitivity of OS cells. Cisplatin-resistant MG63 cells transfected with pcDNA3.0-PTEN received Cisplatin treatment and Cisplatin-resistant MG63 cells as control. (A) Relative protein expression of PTEN was compared between the control group and the pcDNA3.0-PTEN group through western blotting. (B) Quantification of the protein expression of PTEN. (C) Proliferation was measured by CCK-8 assay. (D) Cell apoptosis was detected by flow cytometric analysis. The error bars represent means \pm standard deviation of three independent experiments. ${ }^{*} \mathrm{P}<0.05$ compared with control group and ${ }^{\#} \mathrm{P}<0.05$ compared with Cisplatin group. PTEN, phosphatase and tensin homolog; OS, osteosarcoma; CCK-8, Cell Counting Kit-8.
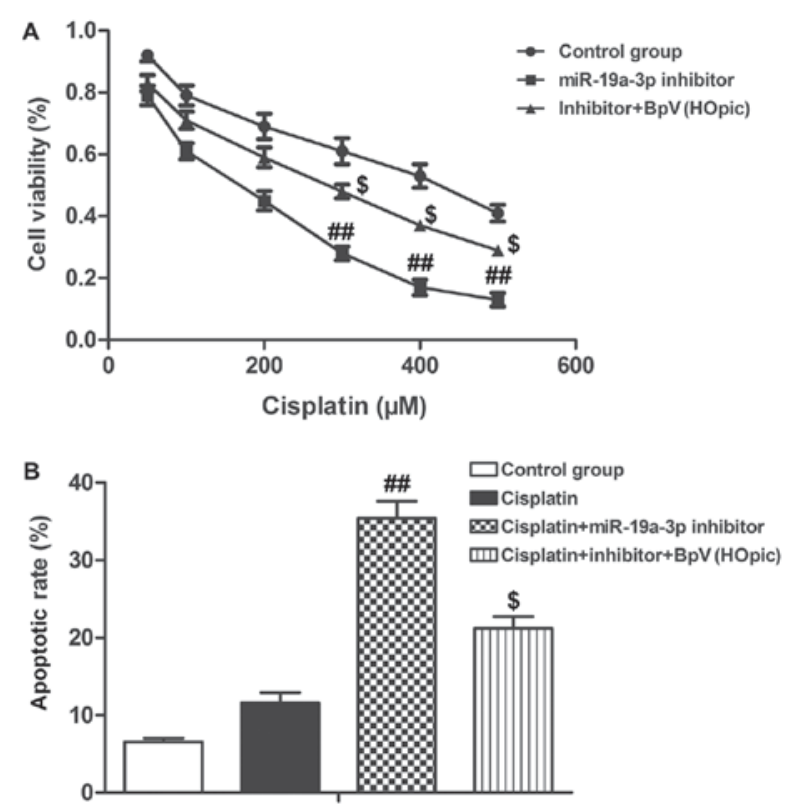

Figure 5. PTEN inhibitor, BpV (HOpic), counteracts the effect of miR-19a-3p inhibitor-transfection on the regulation of chemosensitivity. Cisplatin-resistant MG63 cells transfected with miR-19a-3p inhibitor received Cisplatin treatment with or without $\mathrm{BpV}(\mathrm{HO}$ ic) treatment. (A) Cell proliferation was measured using CCK-8 assay. (B) Cell apoptosis was detected by flow cytometric analysis. The error bars represent the mean \pm standard deviation of three independent experiments. ${ }^{\# \#} \mathrm{P}<0.05$ compared with Cisplatin group and ${ }^{\mathrm{S}} \mathrm{P}<0.05$ compared with Cisplatin + miR-19a-3p inhibitor group. PTEN, phosphatase and tensin homolog; CCK-8, Cell Counting Kit-8. proliferation-associated proteins, including Ki67 and PCNA, compared with the Cisplatin treatment group. Cisplatin treatment combined with miR-19a-3p inhibitor transfection increased the apoptotic rate, increased the expression of Bax, and decreased the expression of Bcl-2 compared with the Cisplatin treatment group. In summary, these results suggest that the silencing of miR-19a-3p enhances chemosensitivity to Cisplatin in OS cells by suppressing cell proliferation and promoting cell apoptosis in treatment with Cisplatin.

PTEN is a tumor suppressor and its expression is often downregulated in various types of tumors $(24,25)$. Similarly, downregulation of the expression of PTEN in OS cells was observed in the present study. Silencing of PTEN has been reported to induce activation of the PI3K/AKT pathway, which leads to cell survival and apoptosis resistance $(26,27)$. Previous studies have reported that PTEN is regulated by miRNAs in many types of cancers. Liu et al (26) indicated that miR-206 inhibited head and neck squamous cell carcinoma (HNSCC) cell progression by targeting HDAC6 via the PTEN/AKT/mTOR pathway. miR-130a increased cell proliferation and tumor growth through repression of PTEN (28). miRNA-21 enhanced chemoresistance to Cisplatin by negatively regulating PTEN in epithelial ovarian cancer (29). The miRNA/PTEN axis may serve an important role in the regulation of tumorigenesis. In the present study, bioinformatics tools demonstrated that the 3'-UTR of the PTEN gene 
harbored a putative binding site for miR-19a-3p. Expression of PTEN was significantly downregulated in the miR-19a-3p mimics group and significantly upregulated in the miR-19a-3p inhibitor group, indicating that the expression of PTEN was negatively regulated by miR-19a-3p. To further examine the interaction between PTEN and miR-19a-3p, a luciferase reporter assay was performed and the results suggested that miR-19a-3p directly targeted PTEN in OS cells. In order to examine the effect of PTEN in the chemosensitivity of OS cells, cispaltin-resistant MG-63 cells were transfected with pcDNA3.0-PTEN to up-regulate the expression of PTEN. The present study revealed that Cisplatin treatment combined with pcDNA3.0-PTEN transfection decreased cell proliferation and increased apoptotic rate compared with Cisplatin treatment alone, suggesting that overexpression of PTEN enhanced chemosensitivity of OS cells to Cisplatin. Therefore, silencing of miR-19a-3p may result in upregulation of the expression of PTEN in OS cells. To verify this result, $\mathrm{Bpv}(\mathrm{HOpic})$, a PTEN inhibitor, was used to treat miR-19a-3p inhibitor-transfected cells. It was observed that inhibition of PTEN upregulated cell proliferation and downregulated apoptotic rate compared with the Cisplatin-treated miR-19a-3p inhibitor group, indicating that inhibition of PTEN counteracted the effect of the miR-19a-3p inhibitor on the chemosensitivity of OS cells.

Taken together, the results of the present study indicate a role of miR-19a-3p in the chemosensitivity of OS cells. miR-19a-3p was overexpressed in OS cell lines and downregulation of miR-19a-3p enhanced chemosensitivity to Cisplatin by elevating the expression of tumor suppressor PTEN in OS cells. Therefore, an miR-19a-3p/PTEN axis may be a potential target for the treatment of OS.

\section{Acknowledgements}

The authors would like to thank members of The First People's Hospital of Wenling, for providing helpful discussion regarding the present study.

\section{Funding}

No funding was received.

\section{Availability of data and materials}

The datasets used and/or analyzed during the current study are available from the corresponding author on reasonable request.

\section{Authors' contributions}

BZ interpreted the main data regarding the cell survival and apoptosis analysis. JNZ was responsible for western blot and statistical analysis. YL was responsible for study design and drafting of the manuscript. All authors read and approved the final manuscript.

\section{Ethics approval and consent to participate}

Not applicable.

\section{Patient consent for publication}

Not applicable.

\section{Competing interests}

The authors declare that they have no competing interests.

\section{References}

1. Arndt CA, Rose PS, Folpe AL and Laack NN: Common musculoskeletal tumors of childhood and adolescence. Mayo Clin Proc 87: 475-487, 2012.

2. Ta HT, Dass CR, Choong PF and Dunstan DE: Osteosarcoma treatment: State of the art. Cancer Metastasis Rev 28: 247-263, 2009.

3. Kempf-Bielack B, Bielack SS, Jurgens H, Branscheid D, Berdel WE, Exner GU, Göbel U, Helmke K, Jundt G, Kabisch $\mathrm{H}$, et al: Osteosarcoma relapse after combined modality therapy: An analysis of unselected patients in the Cooperative Osteosarcoma Study Group (COSS). J Clin Oncol 23: 559-568, 2005.

4. Thompson LD: Osteosarcoma. Ear, Nose Throat J 92: 288-290, 2013.

5. Chang Z, Huo L, Li K, Wu Y and Hu Z: Blocked autophagy by miR-101 enhances osteosarcoma cell chemosensitivity in vitro. ScientificWorldJournal 2014: e794756, 2014.

6. Zhou Y, Huang Z, Wu S, Zang X, Liu M and Shi J: miR-33a is up-regulated in chemoresistant osteosarcoma and promotes osteosarcoma cell resistance to cisplatin by down-regulating TWIST. J Exp Clin Cancer Res 33: 12, 2014.

7. Wang Y and Lee CG: MicroRNA and cancer-focus on apoptosis. J Cell Mol Med 13: 12-23, 2009.

8. Bartel DP: MicroRNAs: Genomics, biogenesis, mechanism, and function. Cell 116: 281-297, 2004.

9. Iorio MV and Croce CM: MicroRNAs in cancer: Small molecules with a huge impact. J Clin Oncol 27: 5848-5856, 2009.

10. Lu C, Xie Z and Peng Q: MiRNA-107 enhances chemosensitivity to paclitaxel by targeting antiapoptotic factor Bcl-w in non small cell lung cancer. Am J Cancer Res 7: 1863-1873, 2017.

11. Zhang J, Qiu W, Xu S, Yu Q, Liu C, Wang Y, Lu A, Zhang J and Lu X1: MicroRNA-625 inhibits the proliferation and increases the chemosensitivity of glioma by directly targeting AKT2. Am J Cancer Res 7: 1835-1849, 2017.

12. Li Y, Luo T, Wang L, Wu J and Guo S: MicroRNA-19a-3p enhances the proliferation and insulin secretion, while it inhibits the apoptosis of pancreatic $\beta$ cells via the inhibition of SOCS3. Int J Mol Med 38: 1515-1524, 2016.

13. Soria JC, Lee HY, Lee JI, Wang L, Issa JP, Kemp BL, Liu DD, Kurie JM, Mao L and Khuri FR: Lack of PTEN expression in non-small cell lung cancer could be related to promoter methylation. Clin Cancer Res 8: 1178-1184, 2002.

14. Livak KJ and Schmittgen TD: Analysis of relative gene expression data using real-time quantitative PCR and the 2(-Delta Delta C(T)) method. Methods 25: 402-408, 2001.

15. Shen J, Niu W, Zhang H, Jun M and Zhang H: Downregulation of MicroRNA-147 Inhibits Cell Proliferation and Increases the Chemosensitivity of Gastric Cancer Cells to 5-Fluorouracil by Directly Targeting PTEN. Oncol Res, doi: 10.3727/096504017X 15061902533715, 2017.

16. Bielack SS, Kempf-Bielack B, Delling G, Exner GU, Flege S, Helmke K, Kotz R, Salzer-Kuntschik M, Werner M, Winkelmann W, etal: Prognostic factors in high-grade osteosarcoma of the extremities or trunk: An analysis of 1,702 patients treated on neoadjuvant cooperative osteosarcoma study group protocols. J Clin Oncol 20: 776-790, 2002.

17. Dasari S and Tchounwou PB: Cisplatin in cancer therapy: Molecular mechanisms of action. Eur J Pharmacol 740: 364-378, 2014.

18. Lei W, Yan C, Ya J, Yong D, Yujun B and Kai L: MiR-199a-3p affects the multi-chemoresistance of osteosarcoma through targeting AK4. BMC cancer 18: 631, 2018.

19. Xie B, Li Y, Zhao R, Xu Y, Wu Y, Wang J, Xia D, Han W and Chen D: Identification of key genes and miRNAs in osteosarcoma patients with chemoresistance by bioinformatics analysis. Biomed Res Int. 2018: 4761064, 2018. 
20. Wang SN, Luo S, Liu C, Piao Z, Gou W, Wang Y, Guan W, Li Q, Zou H, Yang ZZ, et al: miR-491 inhibits osteosarcoma lung metastasis and chemoresistance by targeting alphaB-crystallin. Mol Ther 25: 2140-2149, 2017.

21. Rajabpour A, Afgar A, Mahmoodzadeh H, Radfar JE, Rajaei F and Teimoori-Toolabi L: MiR-608 regulating the expression of ribonucleotide reductase M1 and cytidine deaminase is repressed through induced gemcitabine chemoresistance in pancreatic cancer cells. Cancer Chemother Pharmacol 80: 765-775, 2017.

22. Zou M, Wang F, Gao R, Wu J, Ou Y, Chen X, Wang T, Zhou X, Zhu W,Li P, et al: Autophagy inhibition of hsa-miR-19a-3p/19b-3p by targeting TGF- $\beta$ R II during TGF- $\beta 1$-induced fibrogenesis in human cardiac fibroblasts. Sci Rep 6: 24747, 2016.

23. Zhu M, Huang Z, Zhu D, Zhou X, Shan X, Qi LW, Wu L, Cheng W, Zhu J, Zhang L, et al: A panel of microRNA signature in serum for colorectal cancer diagnosis. Oncotarget 8: 17081-17091, 2017.

24. Lotan TL, Heumann A, Rico SD, Hicks J, Lecksell K, Koop C, Sauter G, Schlomm T and Simon R: PTEN loss detection in prostate cancer: Comparison of PTEN immunohistochemistry and PTEN FISH in a large retrospective prostatectomy cohort. Oncotarget 8: 65566-65576, 2017.
25. Ma J, Guo X, Zhang J, Wu D, Hu X, Li J, Lan Q, Liu Y and Dong W: PTEN gene induces cell invasion and migration via regulating AKT/GSK-3 $/ / \beta$-Catenin signaling pathway in human gastric cancer. Dig Dis Sci 62: 3415-3425, 2017.

26. Liu F, Zhao X, Qian Y, Zhang J, Zhang Y and Yin R: MiR-206 inhibits Head and neck squamous cell carcinoma cell progression by targeting HDAC6 via PTEN/AKT/mTOR pathway. Biomed Pharmacother 96: 229-237, 2017.

27. Ma Y, Zhang P, Gao Y, Fan H, Zhang M and Wu J: Evaluation of AKT phosphorylation and PTEN loss and their correlation with the resistance of rituximab in DLBCL. Int J Clin Exp Pathol 8: 14875-14884, 2015.

28. Wei H, Cui R, Bahr J, Bahr J, Zanesi N, Luo Z, Meng W, Liang G, and Croce CM: miR-130a deregulates PTEN and stimulates tumor growth. Cancer Res 77: 6168-6178, 2017.

29. Yu X, Chen Y, Tian R, Li J, Li H, Lv T and Yao Q: miRNA-21 enhances chemoresistance to cisplatin in epithelial ovarian cancer by negatively regulating PTEN. Oncol Lett 14: 1807-1810, 2017. 